\title{
Optofluidic Bragg Grating Sensor for Monolayer Detection
}

\author{
R. M. Parker, J. C. Gates, P. G. R. Smith, M. C. Grossel.
}

Optoelectronics Research Centre and the School of Chemistry, University of Southampton, Highfield, Southampton, United Kingdom, SO17 1BJ. rmp203@soton.ac.uk

An exposed Bragg grating incorporated into a planar waveguide was used to form a refractive index sensor. The high sensitivity to subtle changes allowed the study of surface functionalisation and binding within a microfluidic system.

(C) 2009 Optical Society of America

OCIS codes: (280.4788) Optical sensing and sensors; (230.7380) Waveguides, channelled.

Planar waveguides can be written with a UV-laser into photosensitized silica to produce a wide range of optical devices. Careful modulation of interfering beams allows Bragg gratings to be directly written into the channel (Figure 1):
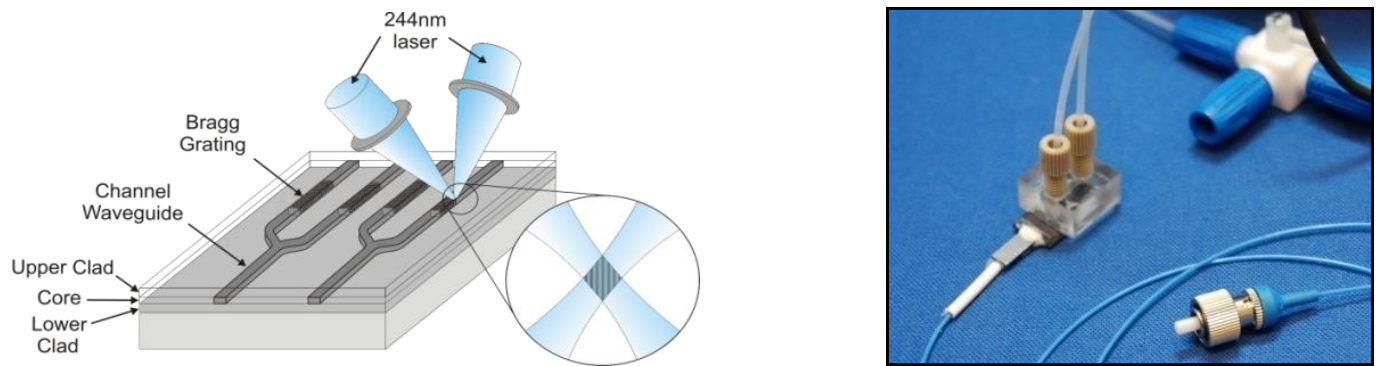

Figure 1 - schematic of direct UV writing of planar waveguides containing Bragg gratings into germanium doped silica wafers (left); and a photograph of the microfluidic cell (right).

These Bragg gratings are inherently sensitive to temperature and strain. However if the surface is etched away, the mode within the grating is exposed to the fluid and becomes sensitive to the refractive index of its surroundings. The corresponding observed shift in Bragg wavelength can be used to detect changes in this environment [1].

The sensitivity of the device to refractive index is dependent on how much of the modal field penetrates into the liquid. Tantalum pentoxide has a significantly higher refractive index (typically 2.08$)$ than that of silica ( 1.44). A thin layer of tantalum pentoxide $(\sim 40 \mathrm{~nm})$ over the etched region of the waveguide pulls the guided mode into the fluid [2]. This increases the proportion of the mode sensitive to the surface region, increasing the sensitivity of the Transverse Electric (TE) mode by up to an order of magnitude (Figure 2.a). In contrast the Transverse Magnetic (TM) mode is not enhanced and this can be used for in situ temperature referencing. Using such a temperature referencing technique significantly improves the robustness of the measurement to thermal drift errors.

a)

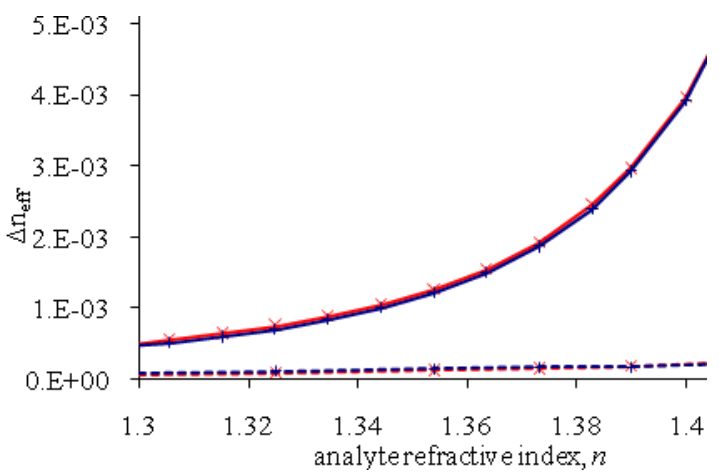

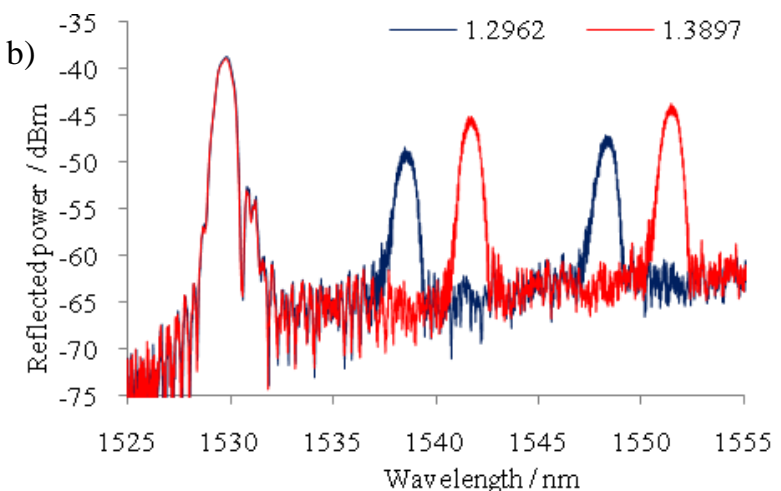

Figure 2.a) - the shift in effective refractive index for a pair of Bragg gratings when exposed to a series of analytes with a range of refractive indices; both before (dashed) and after (solid) deposition of tantalum pentoxide $(40 \mathrm{~nm})$.

Figure 2.b) - the spectral response for analytes with indices of 1.2962 (blue) and 1.3897 (red); showing the shift in the pair of Bragg gratings on change in effective index, compared to the temperature reference grating at $\sim 1530 \mathrm{~nm}$. 
To provide experimental verification of the sensitivity of the planar Bragg sensor, the response of the peak Bragg wavelength to deposition of ultra-thin layers of silica in vacuo onto the sensor was investigated. By measuring within a vacuum system it was possible to avoid surface contamination that could otherwise lead to further thickness changes (Figure 3).

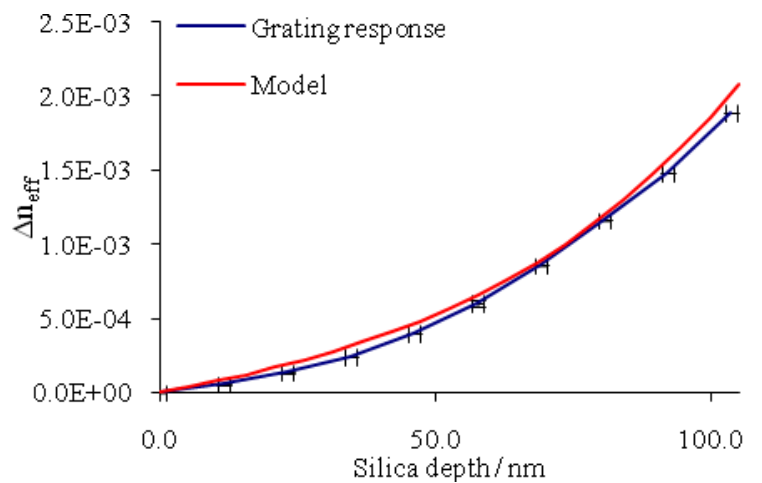

Figure 3 - the effective refractive index shift observed as a function silica deposition onto the sensor surface.

Investigation of the experimental uncertainty indicated a Bragg wavelength shift standard deviation of $1.18 \mathrm{pm}$ and this corresponds to a silica thickness uncertainty of $0.25 \mathrm{~nm}$. This illustrates monolayer sensitivity, albeit in a vacuum environment. The sensitivity is shown to be in the order of $10^{-6}$; an order of magnitude smaller than the shift expected to be observed on tethering a molecular monolayer onto the surface. This allows for the study of surface wetting, functionalisation and ultimately monolayer binding within a microfluidic system. However as the Bragg gratings respond linearly with temperature, with a change of $1{ }^{\circ} \mathrm{C}$ corresponding to a shift of $10 \mathrm{pm}$, it is necessary to control temperature to within $0.01{ }^{\circ} \mathrm{C}$ or, as we shall show, use temperature referencing to compensate.

For practical fluid measurements it is desirable to incorporate the device into a microfluidic system as this offers increased reproducibility and control over that of an open system. Investigation into switching between methanol and isopropanol within a microfluidic system resulted in a reproducible series of changes in the effective index of the grating. However on closer examination it was shown that the previous solvent impacted the following measurement. This has been attributed to the formation and displacement of monolayers on the surface that demonstrate slower kinetics than the bulk fluid, as shown in Figure 4:

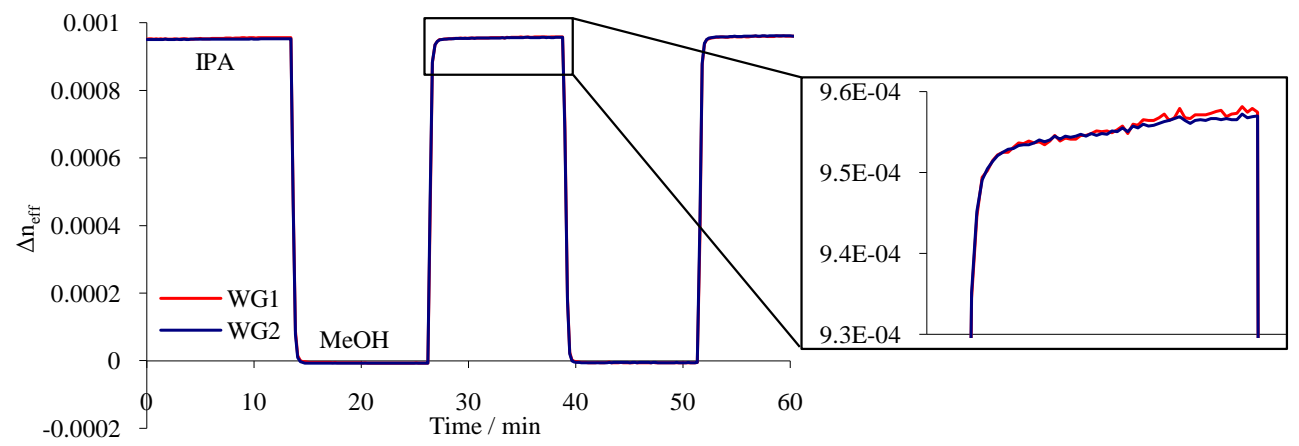

Figure 4 - effective index response to transitions between isopropanol (IPA) and methanol (MeOH) within the microfluidic system; the inset shows the gradual replacement of a surface monolayer of methanol with isopropanol.

We have shown that the device is a highly sensitive refractive index sensor with the potential to detect down to monolayer resolutions for silica deposition and is able to see subtle chemical surface effects. We shall present the latest developments to introduce chemical specifity through host-guest binding and chemical detection.

[1] I.J.G. Sparrow, G. D. Emmerson, C.B.E. Gawith, P.G.R. Smith, M. Kaczmarek, A. Dyadyusha, "First order phase change detection using planar waveguide Bragg grating refractometer", Appl. Phys. B, 81, 1-4, (2005).

[2] G.R. Quigley, R.D. Harris, J.S. Wilkinson, "Sensitivity enhancement of integrated optical sensors by use of thin high-index films", Applied Optics, 38, 6036-6039, (1999). 